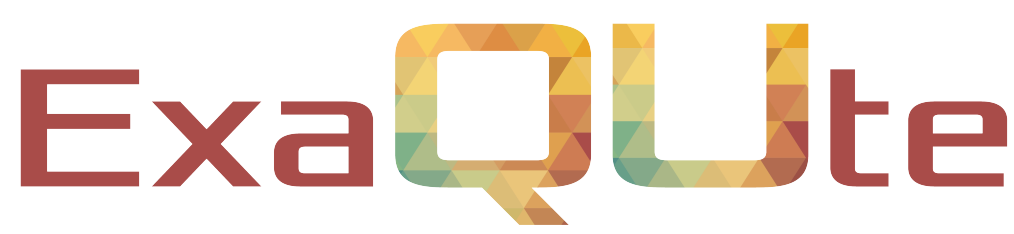

Exascale Quantification of Uncertainties for

Technology and Science Simulation

\title{
First public Release of the solver
}

\section{Document information table}

\begin{tabular}{|l|l|}
\hline Contract number: & 800898 \\
\hline Project acronym: & ExaQUte \\
\hline Project Coordinator: & CIMNE \\
\hline Document Responsible Partner: & CIMNE \\
\hline Deliverable Type: & Software release \\
\hline Dissemination Level: & Public \\
\hline Status: & Final version \\
\hline
\end{tabular}

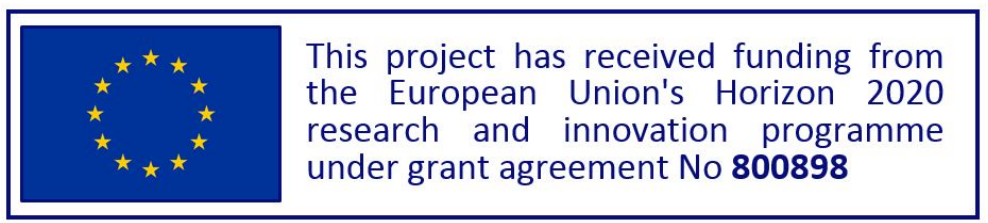




\section{Authoring}

\begin{tabular}{|c|c|c|c|c|}
\hline \multicolumn{5}{|c|}{ Prepared by: CIMNE, BSC and EPFL } \\
\hline Authors & Partner & Modified & Version & Comments \\
\hline Quentin Ayoul-Guilmard & EPFL & \multirow{10}{*}{ All } & \multirow{10}{*}{1.0 .0} & $\mathrm{XMC}$ \\
\hline Rosa M. Badia & $\mathrm{BSC}$ & & & PyCOMPSs \\
\hline Jorge Ejarque & $\mathrm{BSC}$ & & & PyCOMPSs \\
\hline Sundar Ganesh & EPFL & & & $\mathrm{XMC}$ \\
\hline Fabio Nobile & EPFL & & & $\mathrm{XMC}$ \\
\hline Marc Nuñez & CIMNE & & & Kratos Multiphysics \\
\hline Cecilia Soriano & CIMNE & & & Coordination \\
\hline Carlos Roig & CIMNE & & & Kratos Multiphysics \\
\hline Riccardo Rossi & CIMNE & & & Kratos Multiphysics, XMC \\
\hline Riccardo Tosi & CIMNE & & & Kratos Multiphysics, XMC \\
\hline
\end{tabular}

\section{Change Log}

\begin{tabular}{|l|l|l} 
Versions & Modified Page/Sections & Comments
\end{tabular}

\begin{tabular}{l|l|l} 
1.0.0 & All & Submitted version
\end{tabular}

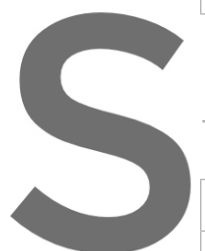

Approval

Aproved by: 


\section{Executive summary}

This deliverable presents the software release of Kratos Multiphysics, together with the XMC library, Hyperloom and PyCOMPSs API definition [8]. This report is meant to serve as a supplement to the public release of the software. Kratos is "a framework for building parallel, multi-disciplinary simulation software, aiming at modularity, extensibility, and high performance. Kratos is written in $\mathrm{C}++$, and counts with an extensive Python interface". XMC is a python library for hierarchical Monte Carlo algorithms. Hyperloom and PyCOMPSs are environments for enabling parallel and distributed computation.
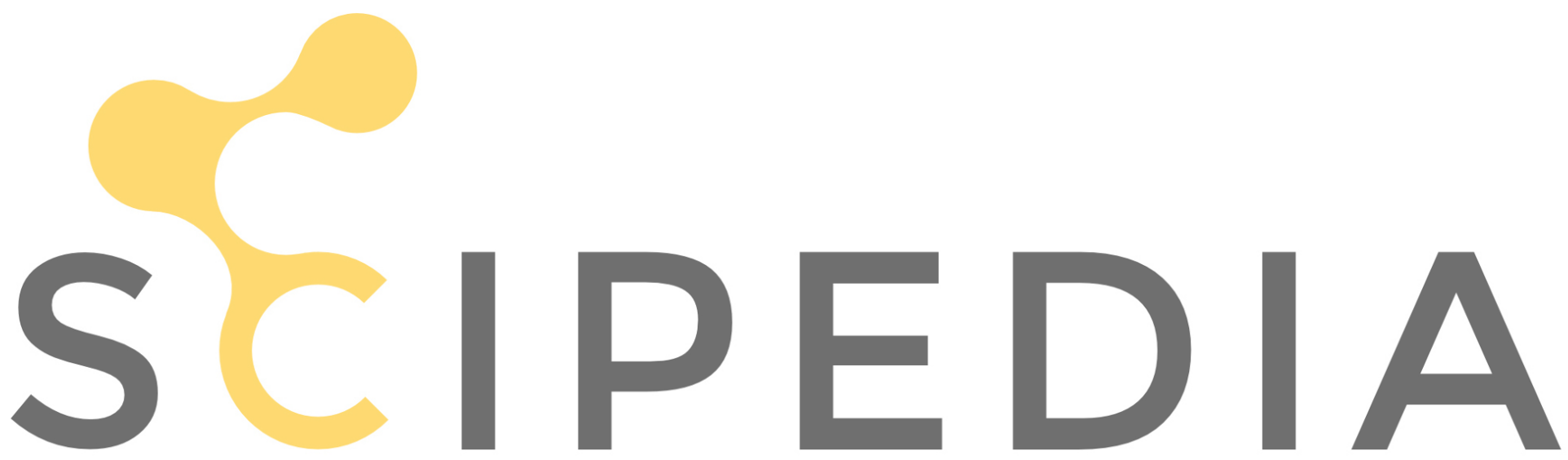

Register for free at https//www.scipedia.com to download the version without the watermark 


\section{Table of contents}

1 Introduction

2 Software structure

3 Examples

4 Current results
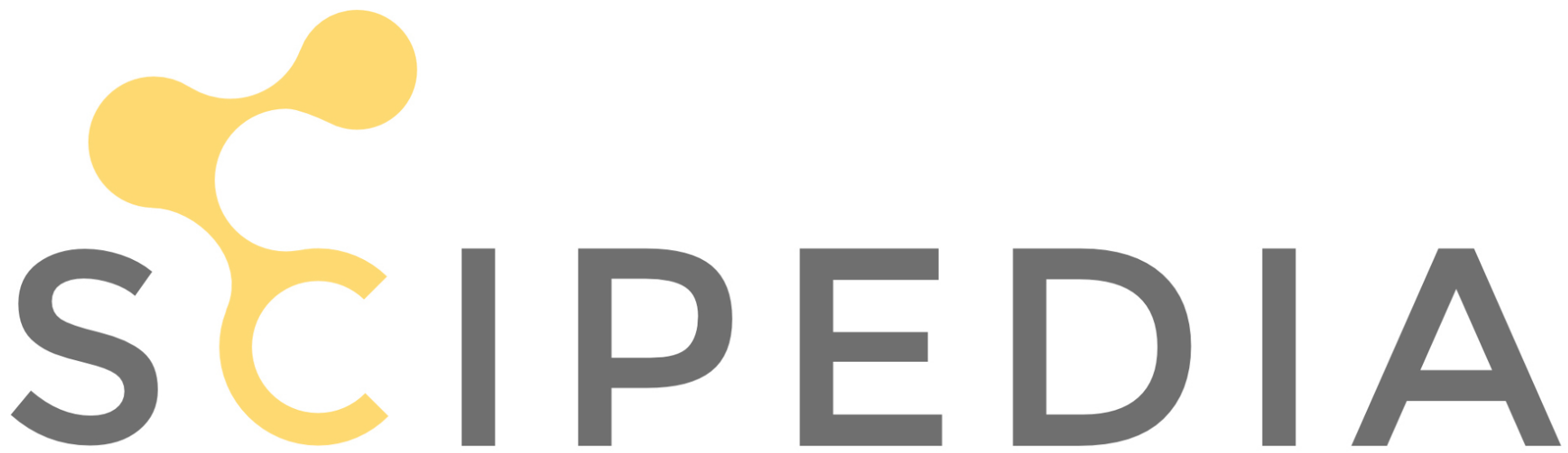

Register for free at https//www.scipedia.com to download the version without the watermark 


\section{Nomenclature / Acronym list}

\begin{tabular}{|l|l|}
\hline Acronym & Meaning \\
\hline API & Application Program Interface \\
\hline Kratos & Kratos MultiPhysics \\
\hline UQ & Uncertainty Quantification \\
\hline
\end{tabular}
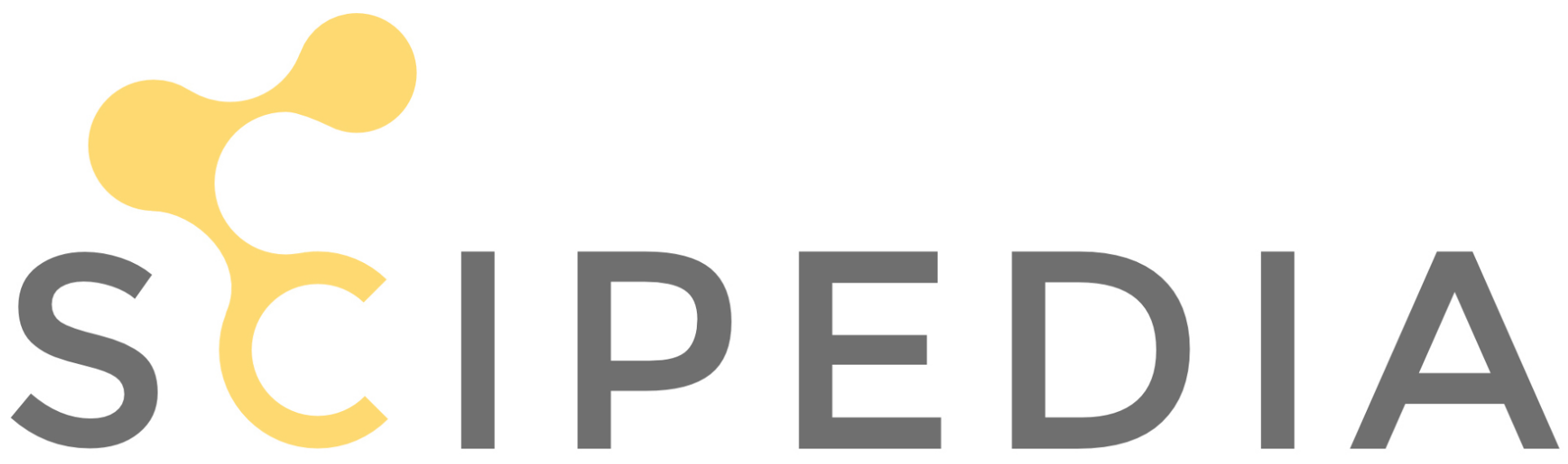

Register for free at https//www.scipedia.com to download the version without the watermark 


\section{Introduction}

The Kratos software [5, 6] is designed for dealing with a multitude of different physical problems, spacing from computational fluid dynamics to convection diffusion, from structural applications to fluid structure interaction. For further details we refer to the Kratos documentation.

One of the most recent capabilities of Kratos is Uncertainty Quantification. UQ is the field of science which studies how uncertainties evolve and propagate in a problem characterized by random parameters, and how the output quantities of interest are affected by the randomness. Even though within the ExaQUte project we are interested in applying UQ to wind engineering problems, where wind velocity is the random quantity, one can easily observe that UQ range of application is much wider, and spaces from engineering to physics and finance. UQ methods have been developed within the XMC library [1], and Kratos is the default solver software of the library. Further details about the XMC library can be found in [2].

Moreover, efficiency is an important factor when dealing with large problems. For this reason, both XMC and Kratos are designed for large scale computing in distributed environments. The scheduling in distributed environments is handled by external libraries: Hyperloom [4] and PyCOMPSs [3, 9, 11]. XMC presents a natural integration with these libraries, making the library partieularly suita
this inner integration of XMC with Hyperloo
the schedulers have been integrated in the so
Therefore, the software release 81 contain
Hyperloom and PyCOMPSS API definitions.
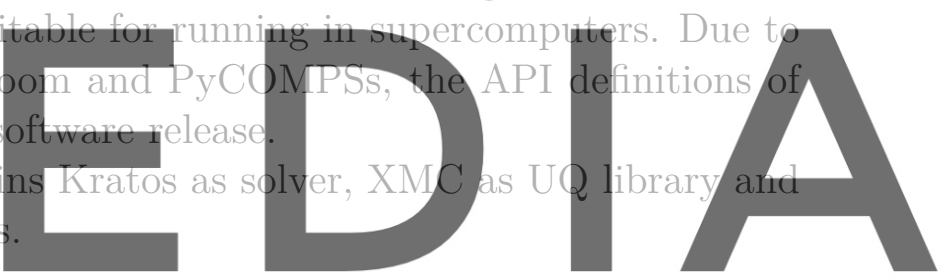

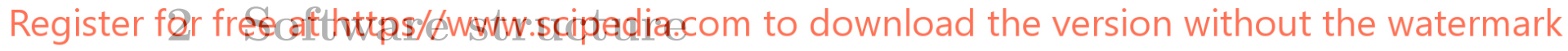

Within Kratos, different applications are organized in different folders. One folder is entirely dedicated to UQ and it is called MultilevelMonteCarloApplication. The folder is located in:

Kratos/applications/MultilevelMonteCarloApplication

This folder contains XMC in:

Kratos/applications/MultilevelMonteCarloApplication/external_libraries/ XMC

and Hyperlooms and PyCOMPSs API definitions in:

Kratos/applications/MultilevelMonteCarloApplication/external_libraries/ PyCOMPSs/exaqute

Examples of usage and tests checking the correct integration between the different libraries are also included.

\section{Examples}

Different algorithms are developed and can be run with this software release: Monte Carlo, Multilevel Monte Carlo, Adaptive Monte Carlo, Adaptive Multilevel Monte Carlo, Con- 
tinuation Multilevel Monte Carlo, Asynchronous Monte Carlo, Asynchronous Multilevel Monte Carlo [10].

Multilevel algorithms are designed to run with different hierarchy strategies: stochastic adaptive refinement, deterministic adaptive refinement or standard uniform refinement. To perform adaptive refinement, the MMG software [7] is exploited for remeshing. To remesh, a metric is a required input to MMG. Different metrics are developed within Kratos, and the one exploited in the examples is based on the hessian of the numerical solution. We refer to the Kratos MMG tutorial and MMG examples for further details.

Examples are located in:

\section{Kratos/applications/MultilevelMonteCarloApplication/external_libraries/ XMC/examples}

These examples are configurable through a JSON file, which is located in the parameters folder (inside the examples folder). Different configuration files are present and available as a reference to run Monte Carlo, Multilevel Monte Carlo or their asynchronous counterparts. For example, to run a standard Monte Carlo example, one should change to the examples folder and execute

\$ python3 run_mc_Kratos.py parameters/

parameters_xmc_test_mc_Kratos_poisson_2d.json

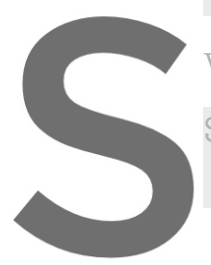
while to run a Multilevel Monte Caxlo example one should execute
\$ python3 run_mlmc_Kratds.py parameters/
parameters_xm__test_nlmc_Kratos_poisson_2d.json
By defautt, such_examples run in serial. If one is interested in running expdoiting distributed computations, Hyperloom and/or PyCOMPSs must be installed in the ma-

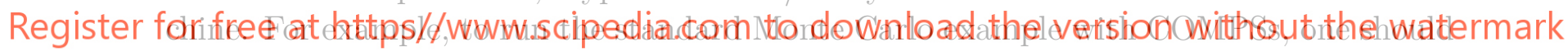
execute:

\section{\$ runcompss --options=value run_mc_Kratos.py parameters/}

parameters_xmc_test_mc_Kratos_poisson_2d.json

However, the user must note that the appropriate imports have to be changed in places marked through XMC from:

\section{from exaqute.ExaquteTaskLocal import *}

to

from exaqute.ExaquteTaskHyperLoom import *

or

from exaqute.ExaquteTaskPyCOMPSs import *

For further details about running in distributed environments, we refer to the MultilevelMonteCarloApplication documentation and to the COMPSs and Kratos tutorial.

\section{Current results}

The work developed and released has been run and tested with several benchmarks, which have been run in different supercomputers. These benchmarks include potential flow, low 
and high Reynolds computational fluid dynamics and structural problems. The software has been tested up to 128 working nodes (6144 CPUs), and showed a very good parallel efficiency. The work developed will be submitted to a peer reviewed journal.

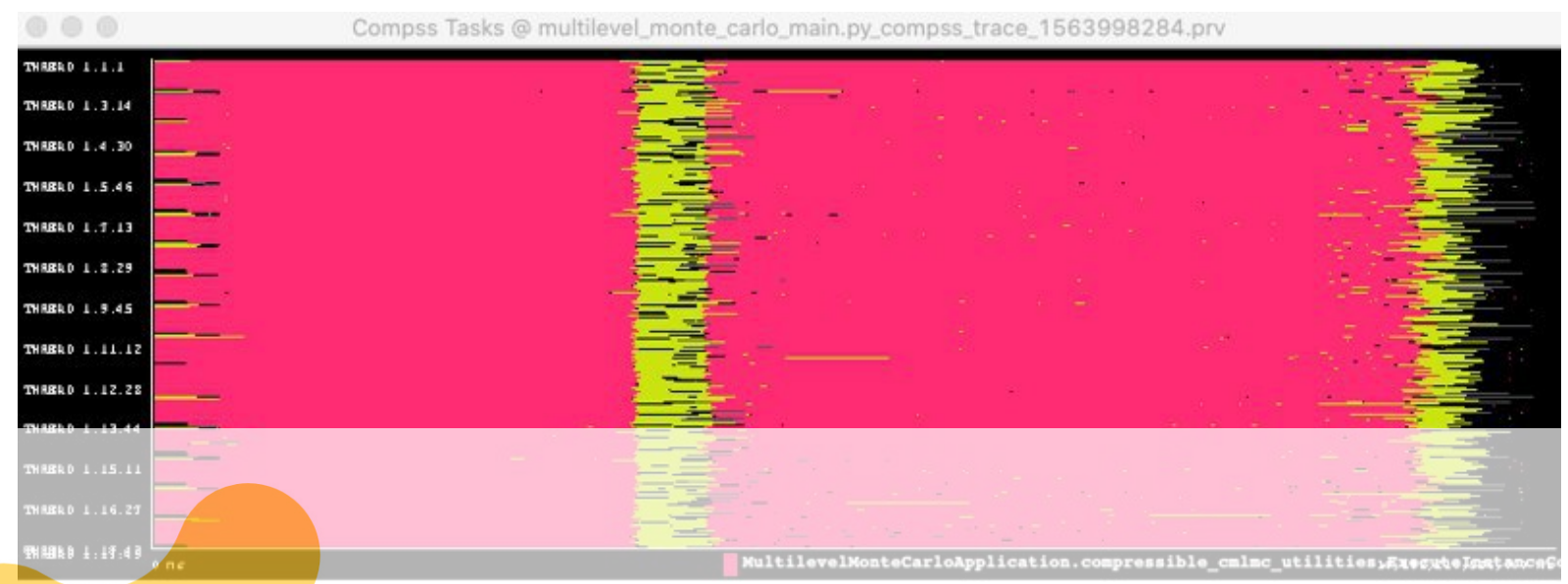

Figure 1: Execution trace of asynchronous Multilevel Monte Carlo algorithm, running with PyCOMPSs.
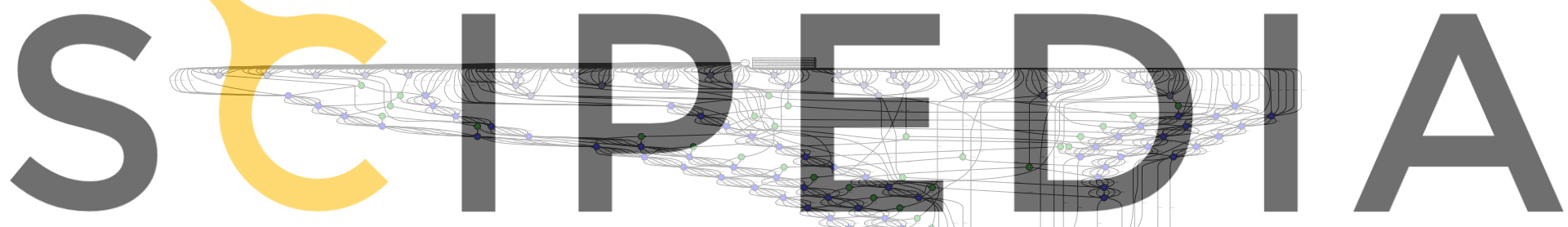

Register for free at https//www.scipedia,com to download the version without the watermark

Figure 2: Graph connection of Multilevel Monte Carlo algorithm dependencies, running with PyCOMPSs. 


\section{References}

[1] R. Amela, Q. Ayoul-Guilmard, R. M. Badia, S. Ganesh, F. Nobile, R. Rossi, and R. Tosi. ExaQUte XMC. May 2019. doi:10.5281/zenodo.3235833.

[2] R. Amela, Q. Ayoul-Guilmard, R. M. Badia, S. Ganesh, F. Nobile, R. Rossi, and R. Tosi. D5.2 Release of ExaQUte MLMC Python engine, May 2019.

[3] R. M. Badia, J. Conejero, C. Diaz, J. Ejarque, D. Lezzi, F. Lordan, C. Ramon-Cortes, and R. Sirvent. COMP superscalar, an interoperable programming framework. SoftwareX, 3-4, 12 2015. doi:10.1016/j.softx.2015.10.004.

[4] V. Cima, S. Böhm, J. Martinovič, J. Dvorskỳ, K. Janurová, T. V. Aa, T. J. Ashby, and V. Chupakhin. Hyperloom: A platform for defining and executing scientific pipelines in distributed environments. In Proceedings of the 9th Workshop and rth Workshop on Parallel Programming and RunTime Management Techniques for Manycore Architectures and Design Tools and Architectures for Multicore Embedded Computing Platforms, pages 1-6. ACM, 2018.

[5] P. Dadvand, R. Rossi, and E. Oñate. An object-oriented environment for developing

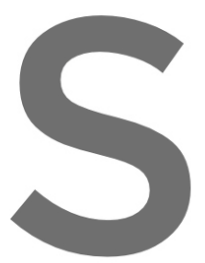
finite element methods in engin
P. Dadvand, R.
sohn, and E.
environments.
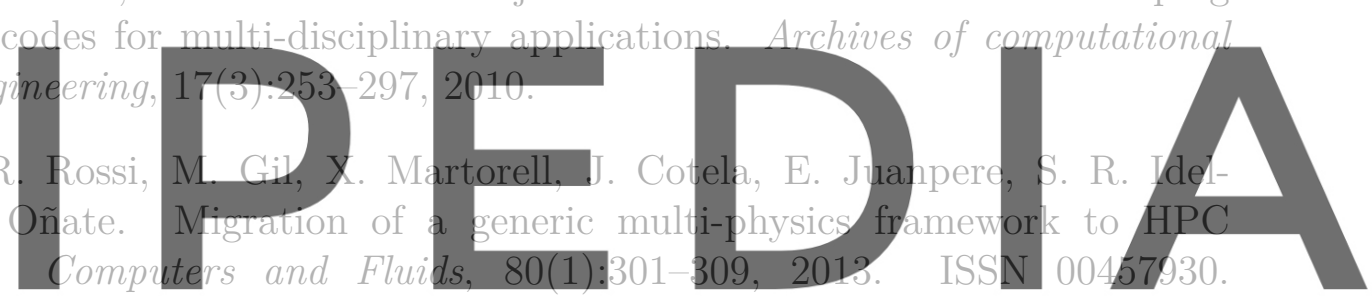

doi:10.1016/j.compfluid.2012.02.004.

Register for free at https/dwww.scipedia.com to download the version without the watermark

7] C. Dapogny, C. Dobrzynski, and P. Frey. Three-dimensional adaptive domain remeshing, implicit domain meshing, and applications to free and moving boundary problems. Journal of Computational Physics, 262:358-378, apr 2014. ISSN 10902716. doi:10.1016/j.jcp.2014.01.005.

[8] V. M. Ferrándiz, P. Bucher, R. Rossi, jcotela, J. Maria, R. Zorrilla, M. A. Celigueta, G. Casas, C. Roig, A. C. Velázquez, P. Dadvand, S. Latorre, J. I. González, I. de Pouplana, miguelmaso, M. Núñez, F. Arrufat, dbaumgaertner, B. Chandra, A. Ghantasala, armingeiser, S. Warnakulasuriya, lluís, J. Gárate, MFusseder, Pablo, AFranci, L. Gracia, thomas, K. B. Sautter, and R. Tosi. KratosMultiphysics/Kratos: Release 8.0, May 2020. URL https://doi.org/10.5281/zenodo.3234644.

[9] F. Lordan, E. Tejedor, J. Ejarque, R. Rafanell, J. Álvarez, F. Marozzo, D. Lezzi, R. Sirvent, D. Talia, and R. M. Badia. ServiceSs: An Interoperable Programming Framework for the Cloud. Journal of Grid Computing, 12(1):67-91, 2014. ISSN 15707873. doi:10.1007/s10723-013-9272-5.

[10] M. Pisaroni, F. Nobile, and P. Leyland. A Continuation Multi Level Monte Carlo (CMLMC) method for uncertainty quantification in compressible inviscid aerodynamics. Computer Methods in Applied Mechanics and Engineering, 326:20-50, 2017. ISSN 00457825. doi:10.1016/j.cma.2017.07.030. 
[11] E. Tejedor, Y. Becerra, G. Alomar, A. Queralt, R. M. Badia, J. Torres, T. Cortes, and J. Labarta. PyCOMPSs: Parallel computational workflows in Python. International Journal of High Performance Computing Applications, 31(1):66-82, 2017. ISSN 17412846. doi:10.1177/1094342015594678.
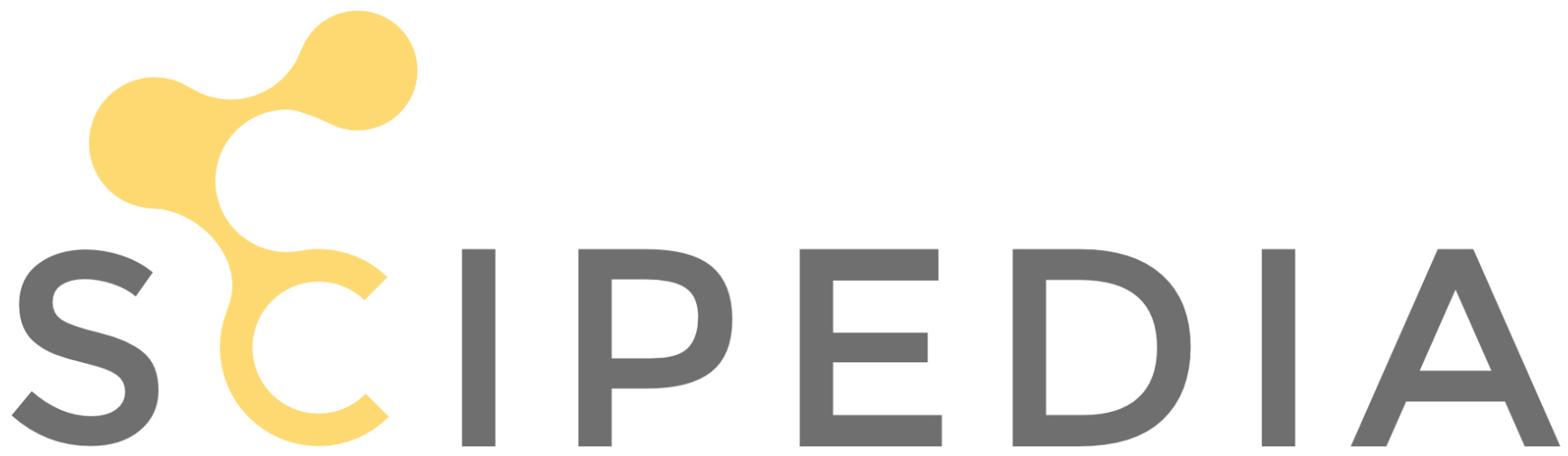

Register for free at https//www.scipedia.com to download the version without the watermark 